\title{
The census of complex organic molecules in the solar type protostar IRAS16293-2422
}

\author{
Ali A. Jaber ${ }^{1,2,3}$, C. Ceccarelli ${ }^{1,2}$, C. Kahane ${ }^{1,2}$, E. Caux ${ }^{4,5}$ \\ Received - ; accepted -
}

\begin{abstract}
Complex Organic Molecules (COMs) are considered crucial molecules, since they are connected with organic chemistry, at the basis of the terrestrial life. More pragmatically, they are molecules in principle difficult to synthetize in the harsh interstellar environments and, therefore, a crucial test for astrochemical models. Current models assume that several COMs are synthesised on the lukewarm grain surfaces $(\gtrsim 30-40 \mathrm{~K})$, and released in the gas phase at dust temperatures $\gtrsim 100 \mathrm{~K}$. However, recent detections of COMs in $\lesssim 20 \mathrm{~K}$ gas demonstrate that we still need important pieces to complete the puzzle of the COMs formation. We present here a complete census of the oxygen and nitrogen bearing COMs, previously detected in different ISM regions, towards the solar type protostar IRAS16293-2422. The census was obtained from the millimeter-submillimeter unbiased spectral survey TIMASSS. Six COMs, out of the 29 searched for, were detected: methyl cyanide, ketene, acetaldehyde, formamide, dimethyl ether, and methyl formate. The multifrequency analysis of the last five COMs provides clear evidence that they are present in the cold $(\lesssim 30 \mathrm{~K})$ envelope of IRAS16293-2422, with abundances $0.03-2 \times 10^{-10}$. Our data do not allow to support the hypothesis that the COMs abundance increases with increasing dust temperature in the cold envelope, as expected if COMs were predominately formed on the lukewarm grain surfaces. Finally, when considering also other ISM sources, we find a strong correlation over five orders of magnitude, between the methyl formate and dimethyl ether and methyl formate and formamide abundances, which may point to a link between these two couples of species, in cold and warm gas.
\end{abstract}

\footnotetext{
${ }^{1}$ Univ. Grenoble Alpes, IPAG, F-38000 Grenoble, France

${ }^{2}$ CNRS, IPAG, F-38000 Grenoble, France

${ }^{3}$ University of AL-Muthana, AL-Muthana, Iraq

${ }^{4}$ Université de Toulouse, UPS-OMP, IRAP, Toulouse, France

${ }^{5}$ CNRS, IRAP, 9 Av. Colonel Roche, BP 44346, 31028 Toulouse Cedex 4, France
} 
Subject headings: ISM: abundances — ISM: molecules — stars: formation

\section{Introduction}

Complex Organic Molecules (COMs), namely organic molecules with more than six atoms (Herbst \& van Dishoeck 2009), have been discovered since more than four decades (Ball et al. 1971; Rubin et al. 1971; Solomon et al. 1971; Brown et al. 1975; Blake et al. 1987). Since some COMs have a prebiotic relevance, they immediately rise a great interest and several models were developed to explain why and how these molecules are formed in space. Those models were based on this two-step process: (i) "mother" (or first generation) species were created during the cold star formation process and frozen into the grain mantles; (ii) "daughter" (or second generation) species were synthesised via gas phase reactions from mother species in the warm $(\gtrsim 200 \mathrm{~K})$ regions where the grain mantles sublimate (Millar et al. 1991; Charnley et al. 1992; Caselli et al. 1993). This two-step paradigm, has enjoyed a great success for about a decade, until new observations towards low mass hot corinos (Ceccarelli et al. 2000; Cazaux et al. 2003) and Galactic Center molecular clouds (RequenaTorres et al. 2007) challenged the assumption that COMs are formed by gas phase reactions. At the same time, new laboratory experiments and theoretical computations revisited and ruled out some gas phase reactions crucial in those models (Horn et al. 2004; Geppert et al. 2007). The attention then moved towards the possibility that grains could act as catalysers and that COMs could form on their surfaces at lukewarm ( $\gtrsim 30-40 \mathrm{~K})$ temperatures (Garrod et al. 2009). However, grain surface chemistry is even more difficult to understand than gas phase chemistry, both from a theoretical and experimental point of view. Let us take the example of methanol, one of the simplest COMs. It is supposed to form on the grain surface via successive hydrogenation of frozen CO (Tielens \& Hagen 1982; Taquet et al. 2012). However, while laboratory experiments claim that this is the case (e.g. (Watanabe \& Kouchi 2002; Pirim et al. 2010), theoretical quantum chemistry computations show that the first and last steps towards the $\mathrm{CH}_{3} \mathrm{OH}$ formation have large (tens of $\mathrm{kCal}$ ) energy barriers impossible to surmount in the cold $(\sim 10 \mathrm{~K})$ cloud conditions (Woon 2002; Marenich \& Boggs 2003; Goumans et al. 2008), where the CO hydrogenation is supposed to occur. To add confusion, recent observations have revealed that some COMs (notably acetaldehyde, methyl formate and dimethyl ether) are found in definitively cold $(\lesssim 20 \mathrm{~K})$ regions (Öberg et al. 2010; Bacmann et al. 2012; Cernicharo et al. 2012), challenging the theory of grain surface formation of COMs.

In this context, we examined the millimeter-submillimeter spectral survey obtained towards the solar type protostar IRAS16293-2422 (hereinafter IRAS16293; Caux et al. 2011) 
with the goal to extract the line emission from all oxygen and nitrogen bearing COMs already detected in the ISM, and to estimate their abundances across its envelope. Our emphasis here is on the abundances in the cold $(\lesssim 50 \mathrm{~K})$ region of the envelope, to provide astrochemical modellers with the first systematic survey of COMs in cold gas.

\section{Source description}

IRAS16293 is a solar type Class 0 protostar in the $\rho$ Ophiuchus star forming region, at a distance of $120 \mathrm{pc}$ (Loinard et al. 2008). It has a bolometric luminosity of $22 \mathrm{~L}_{\odot}$ (Crimier et al. 2010). Given its proximity and brightness, it has been the target of numerous studies that have reconstructed its physical and chemical structure. Briefly, IRAS16293 has a large envelope that extends up to $\sim 6000 \mathrm{AU}$ and that surrounds two sources, named I16293-A and I16293-B in the literature, separated by $\sim 5 "(\sim 600$ AU; Wootten (1989); Mundy et al. (1992)). I16293-A sizes are $\sim 1$ ", whereas I16293-B is unresolved at a scale of $\sim 0.4$ " (Zapata et al. 2013). I16293-A itself is composed of two sources, each one emitting a molecular outflow (Mizuno et al. 1990; Loinard et al. 2013). I16293-B possesses a very compact outflow (Loinard et al. 2013) and is surrounded by infalling gas (Pineda et al. 2012; Zapata et al. 2013). From a chemical point of view, IRAS16293 can be considered as composed of an outer envelope, characterised by low molecular abundances, and a hot corino, where the abundance of many molecules increases by orders of magnitude (Ceccarelli et al. 2000, Schöier et al. 2002; Coutens et al. 2013). The transition between the two regions occurs at $\sim 100 \mathrm{~K}$, the sublimation temperature of the icy grain mantles. In the hot corino, several abundant COMs have been detected (Cazaux et al. 2003).

\section{The data set}

\subsection{Observations}

We used the data from The IRAS16293 Millimeter And Submillimeter Spectral Survey (TIMASSS: http://www-laog.obs.ujf-grenoble.fr/heberges/timasss/; Caux et al. 2011). Briefly, the survey covers the $80-280$ and 328-366 GHz frequency intervals and it has been obtained at the IRAM-30m and JCMT-15m telescopes. The data are publicly available on

the TIMASSS web site. Details on the data reduction and calibration can be found in Caux et al. (2011). We recall here the major features, relevant for this work. The telescope beam depends on the frequency and varies between 9" and 30". The spectral resolution varies between 0.3 and $1.25 \mathrm{MHz}$, corresponding to velocity resolutions between 0.51 and 2.25 
$\mathrm{km} / \mathrm{s}$. The achieved $\mathrm{rms}$ is between 4 and $17 \mathrm{mK}$. Note that it is given in a $1.5 \mathrm{~km} / \mathrm{s}$ bin for observations taken with a velocity resolution $\leq 1.5 \mathrm{~km} / \mathrm{s}$, and in the resolution bin for larger velocity resolutions. The observations are centered on IRAS16293B at $\alpha(2000.0)=16^{h} 32^{m}$ $22^{s} .6, \delta(2000.0)=-24^{\circ} 28^{\prime} 33^{\prime \prime}$. Note that the A and B components are both inside the beam of observations at all frequencies.

\subsection{Species identification}

We searched for lines of all the oxygen and nitrogen bearing COMs already detected in the ISM (as reported in the CDMS database: http://www.astro.uni-koeln.de/cdms/molecules), they are listed in Tab. 1. At this scope, we used the list of identified lines in Caux et al. (2011) and double-checked for possible blending and misidentifications. This was obtained via the publicly available package CASSIS (http://cassis.irap.omp.eu), and the CDMS (Müller et al. 2005) and JPL (Pickett et al. 1998) databases. References to the specific articles on the laboratory data of the detected species are Guarnieri \& Huckauf (2003); Kleiner et al. (1996); Neustock et al. (1990); Maeda et al. (2008). In case of doubt on the line identification or in case of presence of important residual baseline effects, we did not consider the relevant line. Except for those few $(\leq 10 \%)$ cases, we used the line parameters (flux, linewidth, rest velocity) in Caux et al. (2011). With these tight criteria, we secured the detection of six COMs: ketene ( $\mathrm{H}_{2} \mathrm{CCO}$ : 13 lines), acetaldehyde $\left(\mathrm{CH}_{3} \mathrm{CHO}: 130\right.$ lines), formamide ( $\mathrm{NH}_{2} \mathrm{CHO}: 17$ lines), dimethyl ether $\left(\mathrm{CH}_{3} \mathrm{OCH}_{3}\right.$ : 65 lines), methyl formate $\left(\mathrm{HCOOCH}_{3}\right.$ : 121 lines) and methyl cyanide $\left(\mathrm{CH}_{3} \mathrm{CN}\right.$ : 38 lines). For comparison, Cazaux et al. (2003) detected $5 \mathrm{CH}_{3} \mathrm{CHO}$ lines, $7 \mathrm{CH}_{3} \mathrm{OCH}_{3}$ lines, and $20 \mathrm{CH}_{3} \mathrm{CHO}$ lines. We do not confirm the Cazaux et al. (2003) detection of acetic acid $\left(\mathrm{CH}_{3} \mathrm{COOH}\right)$ and formic acid ( $\left.\mathrm{HCOOH}\right)$, where these authors reported the possible detection of 1 and 2 lines respectively, none of them in the TIMASSS observed frequency range.

\section{Analysis and results}

\subsection{Model description}

Our goal is to estimate the abundance of the detected COMs across the envelope of IRAS16293, with particular emphasis on the cold envelope (see Introduction). For that, we used the Spectral Line Energy Distribution (SLED) of the detected COMs, and the package GRAPES (GRenoble Analysis of Protostellar Envelope Spectra), based on the code described in Ceccarelli et al. (1996, 2003). Briefly, GRAPES (i) computes the species SLED from a 
spherical infalling envelope with a given structure; (ii) it solves locally the level population statistical equilibrium equations in the beta escape formalism, consistently computing the line optical depth by integrating it over the solid angle at each point of the envelope; (iii) the predicted line flux is then integrated over the whole envelope after convolution with the telescope beam. The abundance $X$ of the considered species is assumed to vary as a function of the radius with a power law in the cold part of the envelope and to jump to a new abundance in the warm part. The transition between the two regions is set by the dust temperature, to simulate the sublimation of the ice mantles, and occurs at $T_{\text {jump }}$. It holds:

$$
\begin{array}{rlrl}
X(r) & =X_{\text {out }}\left(\frac{r}{R_{\text {max }}}\right)^{\alpha} & & \leq T_{\text {jump }} \\
X(r) & =X_{\text {in }} & >T_{\text {jump }}
\end{array}
$$

GRAPES allows us to run large grids of models varying the four parameters, $X_{\text {in }}, X_{\text {out }}$, $\alpha$ and $T_{\text {jump }}$, and to find the best fit to the observed fluxes.

This code has disadvantages and advantages with respect to other codes. The first and obvious disadvantage is that the spherical assumption just holds for the large scale ( $\gtrsim 10$ ": see Crimier et al. 2010) envelope of IRAS16293. At small scales, the presence of the binary system (\$2) makes the spherical symmetry assumption wrong. Consequently, the GRAPES code is, by definition, unable to correctly estimate the emission from the two sources I16293A and I16293-B separately. The derived inner envelope abundance, therefore, is likely a rough indication of the real abundance of the species towards I16293-A and I16293-B. The other disavantage of GRAPES is that it relies on the analysis of the SLED and not on the line profiles. Since the majority of the TIMASSS spectra have a relatively poor spectral resolution ( $\gtrsim 1 \mathrm{~km} / \mathrm{s}$ ), this is appropriate in this case. The great advantage of GRAPES, and the reason why we used it here, is that it is very fast, so that a large multi-parameter space can be explored.

In the specific case of this work, we used the physical structure of the envelope of IRAS16293 as derived by Crimier et al. (2010), which is based on single dish and interferometric continuum observations. Collisional coefficients are only available for methyl cyanide, and not for the other five detected COMs. Since methyl cyanide is a top symmetric molecule, it represents a "particular case" with respect to the other detected COMs, so that, in order to have an homogeneous dataset, we decided to analyse here only the latter molecules, and assume LTE for their level populations. The analysis of the $\mathrm{CH}_{3} \mathrm{CN}$ molecule will be the focus of a future article. Since the density of the IRAS16293 envelope is relatively high (e.g. $5 \times 10^{6} \mathrm{~cm}^{-3}$ at a radius of $870 \mathrm{AU}$, equivalent to 15 " in diameter), we expect that the abundances derived in the LTE approximation are only moderately underestimated. 


\subsection{Results}

For each of the five analysed COMs, we run a large grid of models with the following strategy. We explored the $X_{i n}-X_{\text {out }}$ parameter space (in general we obtained grids of more than $20 \times 20$ ) for $\alpha$ equal to $-1,0$ and +1 , and varied $T_{\text {jump }}$ from 10 to $120 \mathrm{~K}$ by steps of $10 \mathrm{~K}$. Note that we first started with a 3 or 4 orders of magnitude range in $X_{\text {in }}$ and $X_{\text {out }}$ respectively to find a first approximate solution and then we fine-tuned the grid around it. In total, therefore, we run more than $3 \times 10^{4}$ models for each species. The results of the best fit procedure are reported in Tab. 1. Figure 1 shows the example of acetaldehyde. Note that the lines are predicted to be optically thin by the best fit models of all five molecules.

First, we did not find a significant difference in the $\chi^{2}$ best fit value if $\alpha$ is $-1,0$ or +1 , in any of the five COMs, so that Tab. 1 reports the values obtained with $\alpha=0$ only. Second, the $T_{\text {jump }}$ is different in the five COMs: it is $\sim 20 \mathrm{~K}$ for ketene, $\sim 70-80 \mathrm{~K}$ for acetaldehyde and formamide, and $\sim 50 \mathrm{~K}$ for dimethyl ether and methyl formate. Third, the abundance in the outer envelope ranges from $\sim 3 \times 10^{-12}$ to $\sim 2 \times 10^{-10}$ : acetaldehyde and dimethyl ether have the largest values, formamide the lowest, and ketene and methyl formate intermediate values. Fourth, the abundance jumps by about a factor 100 in all COMs

except ketene, which remains practically constant (when the errors are considered). Note that we find a warm envelope abundance of acetaldehyde, dimethyl ether and methyl formate about 10 times smaller than those quoted by Cazaux et al. (2003). The difference mostly derives from a combination of different $T_{\text {jump }}$ (assumed $100 \mathrm{~K}$ in Cazaux et al.(2003)), which implies different emitting sizes, and a different $\mathrm{H}_{2}$ column density. As also emphasised by Cazaux et al. (2003), their hot corino sizes and $\mathrm{H}_{2}$ column density were best guessed and, consequently, uncertain, whereas in the present work they are self-consistently estimated from the molecular lines.

Finally, for the undetected species we derived the upper limits to the abundance in the outer (assuming $\mathrm{N}\left(\mathrm{H}_{2}\right)=8 \times 10^{22} \mathrm{~cm}^{-2}$, diameter=30", $\mathrm{T}=20 \mathrm{~K}$ ) and inner (assuming $\mathrm{N}\left(\mathrm{H}_{2}\right)=3 \times 10^{23} \mathrm{~cm}^{-2}$, diameter $\left.=3 ", \mathrm{~T}=60 \mathrm{~K}\right)$ envelope listed in Tab. 1.

\section{Discussion}

The analysis of outer and inner abundances of the five detected COMs leads to three major considerations and results.

\section{COMs in the cold envelope:}

The first important result of this analysis is the presence of COMs in the cold part of the envelope, with an abundance approximately constant. This is the first time that we have 
unambiguous evidence that also the cold outer envelope of (low mass) protostars can host COMs. Bacmann et al. (2012) reported the detection of acetaldehyde, dimethyl ether and methyl formate with abundances around $10^{-11}$ (with an uncertainty of about one order of magnitude) towards a cold $(\lesssim 10 \mathrm{~K})$ pre-stellar core. Öberg et al. (2010); Cernicharo et al. (2012) reported the detection of the same molecules in B1-b, a low mass protostar where the temperature of the emitting gas is estimated $12-15 \mathrm{~K}$ (but no specific analysis to separate possible emission from warm gas has been carried out in this case), with similar abundances. In the cold envelope of IRAS16293, these COMs seem to be slightly more abundant, with abundances around $10^{-10}$, possibly because the gas is slightly warmer. If the dust surface chemistry dominated the formation of COMs in the outer envelope, the COM abundance would increase with increasing dust temperature, namely decreasing radius in the cold envelope. However, our analysis does not show a definitively better $\chi^{2}$ for the solution corresponding to $\alpha=-1$, so that it cannot support this hypothesis. These new measurements add evidence that COMs, at least the ones studied here, are possibly formed also in cold conditions in addition to the warm grain surfaces, as predicted by current models (see Introduction).

\section{Comparison with other objects:}

Additional information on the formation (and destruction) routes of the detected COMs can be gained by the comparison of the COM abundances in galactic objects with different conditions (temperature, density and history) and Solar System comets. We consider here the abundances normalised to that of methyl formate, a molecule which has been detected in all objects that we want to compare. Figure 2 graphically shows this comparison. Ketene seems to be the most sensitive species in distinguishing two groups of objects: "cold" objects, formed by the cold and Galactic Center clouds, and the outer envelope of IRAS16293, and "warm" objects, constituted by the IRAS16293 hot corino (the only hot corino where the five COMs of this study have been detected so far) and the massive hot cores. In the first group, ketene has an abundance larger than $\sim 0.1$ with respect to methyl formate. In the second group, the relative abundance is lower than $\sim 0.1$. Finally, comets are definitively different from the hot cores, which are often compared with in the literature (see also the discussion in Caselli \& Ceccarelli 2012).

\section{Correlations vs methyl formate.}

Figure 3 shows the abundance of dimethyl ether, formamide, acetaldehyde and ketene as a function of the abundance of methyl formate in different ISM sources. The linear correlation between the methyl formate and dimethyl ether is striking (Pearson correlation coefficient equal to 0.95 and power law index equal to 1.0). It covers almost five orders of magnitude, so that it persists even considering the dispersion of the measurements and the uncertainty linked to the determination of the absolute abundances mentioned above. This linear cor- 
relation, previously observed over a smaller range (e.g.Brouillet et al. (2013)), gives us an important and remarkable message: probably the precursor of methyl formate and dimethyl ether is either the same (Brouillet et al. 2013) or one of the two is the precursor of the other, an hypothesis that has not been invoked in the literature so far. We can not rule out other explanations, but they seem less likely at this stage. The bottom line is that such a link between these two species must be the same in cold and warm gas. This does not favour a formation mechanism of these two COMs on the grain surfaces for, according to the existing models, the mechanism does not work at low temperatures. Current chemical networks (e.g. KIDA at http://kida.obs.u-bordeaux1.fr and UMIST at http://www.udfa.net) do not report reactions linking the two species. Also the recent article by Vasyunin \& Herbst (2013), which proposes new reactions for explaining the Bacmann et al. (2012) and Cernicharo et al. (2012) observations, does not suggest a link between methyl formate and dimethyl ether. We suggest here that those networks are missing this important piece.

Similar analysis and conclusion (Pearson correlation coefficient equal to 0.92 and power law index equal to 0.8) apply to the methyl formate and formamide. On the contrary, the correlation between methyl formate and acetaldehyde or ketene is poorer (Pearson correlation coefficient equal to 0.66 and 0.62 , power law index equal to 0.5 and 0.4 , respectively).

\section{Conclusions}

We searched for all oxygen and nitrogen bearing COMs observed in the ISM, towards the envelope of IRAS16293. We detected six COMs: methyl cyanide, ketene, acetaldehyde, formamide, dimethyl ether, and methyl formate. We report the analysis of the last five species. A specific analysis of methyl cyanide emission will be presented in a subsequent paper. For each species, several lines covering a large upper level energy range (up to 150 $\mathrm{K}$ ) are detected. This allows us to disentangle the emission originating in the cold and warm envelope, respectively, and where the transition between the two occurs. The main results of this study can be summarised in three points:

1- The five analysed COMs are all present in the cold envelope of IRAS16293. Acetaldehyde and dimethyl ether have the largest abundances, $\sim 10^{-10}$, slightly larger than the values found in other cold objects (Bacmann et al. 2012; Cernicharo et al. 2012). These new measurements add support to the idea that a relatively efficient formation mechanism for these COMs must exist in the cold gas phase.

2- When considering the abundance of the five analysed COMs, the ketene abundance relative to methyl formate is different in cold and hot objects. Besides, comets are different from the hot cores. 
3- There is a remarkable correlation between the abundance of methyl formate and that of dimethyl ether and formamide. The correlation spans over five orders of magnitude. This may suggest that both dimethyl ether and formamide have a progenitor common with methyl formate, and that the mechanism of their formation is gas phase reactions. We suggest that the current chemical networks still miss important pieces.

We thank an anonymous referee for useful comments that helped to improve the article. This work has been supported by l'Agence Nationale pour la Recherche (ANR), France (project FORCOMS, contracts ANR-08-BLAN-0225). We acknowledge the financial support

from the university of Al-Muthana and ministry of higher education and scientific research in Iraq .

\section{REFERENCES}

Bacmann, A., Taquet, V., Faure, A., Kahane, C., \& Ceccarelli, C. 2012, A\&A, 541, L12

Ball, J. A., Gottlieb, C. A., Lilley, A. E., \& Radford, H. E. 1971, IAU Circ., 2350, 1

Blake, G. A., Sutton, E. C., Masson, C. R., \& Phillips, T. G. 1987, ApJ, 315, 621

Brouillet, N., Despois, D., Baudry, A., et al. 2013, A\&A, 550, A46

Brown, R. D., Crofts, J. G., Godfrey, P. D., et al. 1975, ApJ, 197, L29

Caselli, P., Hasegawa, T. I., \& Herbst, E. 1993, ApJ, 408, 548

Caux, E., Kahane, C., Castets, A., et al. 2011, A\&A, 532, A23

Cazaux, S., Tielens, A. G. G. M., Ceccarelli, C., et al. 2003, ApJ, 593, L51

Ceccarelli, C., Castets, A., Caux, E., et al. 2000, A\&A, 355, 1129

Ceccarelli, C., Hollenbach, D. J., \& Tielens, A. G. G. M. 1996, ApJ, 471, 400

Ceccarelli, C., Maret, S., Tielens, A. G. G. M., Castets, A., \& Caux, E. 2003, A\&A, 410, 587

Cernicharo, J., Marcelino, N., Roueff, E., et al. 2012, ApJ, 759, L43

Charnley, S. B., Tielens, A. G. G. M., \& Millar, T. J. 1992, ApJ, 399, L71

Coutens, A., Vastel, C., Cabrit, S., et al. 2013, A\&A, 560, A39 
Crimier, N., Ceccarelli, C., Maret, S., et al. 2010, A\&A, 519, A65

Garrod, R. T., Vasyunin, A. I., Semenov, D. A., Wiebe, D. S., \& Henning, T. 2009, ApJ, 700, L43

Geppert, W. D., Vigren, E., Hamberg, M., et al. 2007, in European Planetary Science Congress 2007, 613

Goumans, T. P. M., Catlow, C. R. A., \& Brown, W. A. 2008, The Journal of Chemical Physics, 128,

Guarnieri, A., \& Huckauf, A. 2003, Naturforsch, 58, 275

Herbst, E., \& van Dishoeck, E. F. 2009, ARA\&A, 47, 427

Horn, A., Møllendal, H., Sekiguchi, O., et al. 2004, ApJ, 611, 605

Kleiner, I., Lovas, F. J., \& Godefroid, M. 1996, Journal of Physical and Chemical Reference Data, 25, 1113

Loinard, L., Torres, R. M., Mioduszewski, A. J., \& Rodríguez, L. F. 2008, ApJ, 675, L29

Loinard, L., Zapata, L. A., Rodríguez, L. F., et al. 2013, MNRAS, 430, L10

Maeda, A., De Lucia, F. C., \& Herbst, E. 2008, Journal of Molecular Spectroscopy, 251, 293

Marenich, A., \& Boggs, J. 2003, The Journal of Chemical Physics, 107, 2343

Millar, T. J., Herbst, E., \& Charnley, S. B. 1991, ApJ, 369, 147

Mizuno, A., Fukui, Y., Iwata, T., Nozawa, S., \& Takano, T. 1990, ApJ, 356, 184

Müller, H. S. P., Schlöder, F., Stutzki, J., \& Winnewisser, G. 2005, Journal of Molecular Structure, 742, 215

Mundy, L. G., Wootten, A., Wilking, B. A., Blake, G. A., \& Sargent, A. I. 1992, ApJ, 385, 306

Neustock, W., Guarnieri, A., \& G, D. 1990, Naturforsch, 45, 702

Öberg, K. I., Bottinelli, S., Jørgensen, J. K., \& van Dishoeck, E. F. 2010, ApJ, 716, 825

Pickett, H. M., Poynter, R. L., Cohen, E. A., et al. 1998, J. Quant. Spec. Radiat. Transf., 60,883 
Pineda, J. E., Maury, A. J., Fuller, G. A., et al. 2012, A\&A, 544, L7

Pirim, C., Krim, L., \& Laffon, C. 2010, The Journal of Chemical Physics, 114, 3320

Requena-Torres, M. A., Martin-Pintado, J., Martin, S., \& Amo-Baladron, A. 2007, ATNF Proposal, 939

Rubin, R. H., Swenson, Jr., G. W., Benson, R. C., Tigelaar, H. L., \& Flygare, W. H. 1971, ApJ, 169, L39

Schöier, F. L., Jørgensen, J. K., van Dishoeck, E. F., \& Blake, G. A. 2002, A\&A, 390, 1001

Solomon, P. M., Jefferts, K. B., Penzias, A. A., \& Wilson, R. W. 1971, ApJ, 168, L107

Taquet, V., Ceccarelli, C., \& Kahane, C. 2012, A\&A, 538, A42

Tielens, A. G. G. M., \& Hagen, W. 1982, A\&A, 114, 245

Watanabe, N., \& Kouchi, A. 2002, ApJ, 571, L173

Woon, D. E. 2002, ApJ, 571, L177

Wootten, A. 1989, ApJ, 337, 858

Zapata, L. A., Loinard, L., Rodríguez, L. F., et al. 2013, ApJ, 764, L14 
Table 1: Results of the analysis.

\begin{tabular}{|c|c|c|c|c|c|c|c|c|}
\hline Species & Formula & $\begin{array}{c}\mathrm{X}_{i n} \\
{\left[10^{-8}\right]}\end{array}$ & $\begin{array}{c}\mathrm{X}_{\text {out }} \\
{\left[10^{-10}\right]}\end{array}$ & $\begin{array}{c}T_{\text {jump }} \\
{[\mathrm{K}]}\end{array}$ & $\mathrm{DF}$ & $\chi^{2}$ & $\begin{array}{c}\text { Radius } \\
{[\mathrm{AU}]}\end{array}$ & $\begin{array}{c}\text { Size } \\
\text { ["] }\end{array}$ \\
\hline \multicolumn{9}{|c|}{ Detected COMs } \\
\hline Ketene & $\mathrm{H}_{2} \mathrm{CCO}$ & $0.01 \pm 0.005$ & $0.3 \pm 0.08$ & $20_{-5}^{+20}$ & 10 & 0.63 & 1800 & 31 \\
\hline Acetaldehyde & $\mathrm{CH}_{3} \mathrm{CHO}$ & $0.3 \pm 0.2$ & $1 \pm 0.2$ & $70 \pm 5$ & 127 & 0.79 & 127 & 2 \\
\hline Formamide & $\mathrm{NH}_{2} \mathrm{CHO}$ & $0.06 \pm 0.02$ & $0.03 \pm 0.02$ & $80 \pm 5$ & 14 & 0.69 & 100 & 2 \\
\hline Dimethyl ether & $\mathrm{CH}_{3} \mathrm{OCH}_{3}$ & $4 \pm 1$ & $2 \pm 1$ & $50 \pm 10$ & 62 & 0.72 & 240 & 4 \\
\hline Methyl formate & $\mathrm{HCOOCH}_{3}$ & $0.9 \pm 0.2$ & $0.3 \pm 0.1$ & $50 \pm 5$ & 118 & 0.78 & 240 & 4 \\
\hline \multicolumn{9}{|c|}{ Undetected COMs } \\
\hline Ethylene oxide & $\mathrm{c}-\mathrm{C}_{2} \mathrm{H}_{4} \mathrm{O}$ & $\lesssim 0.1$ & $\lesssim 3$ & & & & & \\
\hline Vinyl alcohol & $\mathrm{H}_{2} \mathrm{CCHOH}$ & $\lesssim 0.04$ & $\lesssim 1$ & & & & & \\
\hline Ethanol & $\mathrm{C}_{2} \mathrm{H}_{5} \mathrm{OH}$ & $\lesssim 0.5$ & $\lesssim 8$ & & & & & \\
\hline Formic acid & $\mathrm{HCOOH}$ & $\lesssim 0.03$ & $\lesssim 0.8$ & & & & & \\
\hline Propynal & $\mathrm{HC}_{2} \mathrm{CHO}$ & $\lesssim 0.02$ & $\lesssim 0.5$ & & & & & \\
\hline Cyclopropenone & $\mathrm{c}-\mathrm{H}_{2} \mathrm{C}_{3} \mathrm{O}$ & $\lesssim 0.004$ & $\lesssim 0.1$ & & & & & \\
\hline Acrolein & $\mathrm{C}_{2} \mathrm{H}_{3} \mathrm{CHO}$ & $\lesssim 0.02$ & $\lesssim 0.6$ & & & & & \\
\hline Acetone & $\mathrm{CH}_{3} \mathrm{COCH}_{3}$ & $\lesssim 0.07$ & $\lesssim 2$ & & & & & \\
\hline Propanal & $\mathrm{CH}_{3} \mathrm{CH}_{2} \mathrm{CHO}$ & $\lesssim 0.1$ & $\lesssim 2$ & & & & & \\
\hline Glycolaldehyde & $\mathrm{CH}_{2}(\mathrm{OH}) \mathrm{CHO}$ & $\lesssim 0.1$ & $\lesssim 3$ & & & & & \\
\hline Ethyl methyl ether & $\mathrm{C}_{2} \mathrm{H}_{5} \mathrm{OCH}_{3}$ & $\lesssim 0.5$ & $\lesssim 9$ & & & & & \\
\hline Ethyleneglycol & $\left(\mathrm{CH}_{2} \mathrm{OH}\right)_{2}$ & $\lesssim 0.2$ & $\lesssim 5$ & & & & & \\
\hline Ethyl formate & $\mathrm{C}_{2} \mathrm{H}_{5} \mathrm{OCHO}$ & $\lesssim 0.2$ & $\lesssim 5$ & & & & & \\
\hline Methylamine & $\mathrm{CH}_{3} \mathrm{NH}_{2}$ & $\lesssim 0.1$ & $\lesssim 3$ & & & & & \\
\hline Methylisocyanide & $\mathrm{CH}_{3} \mathrm{NC}$ & $\lesssim 0.002$ & $\lesssim 0.07$ & & & & & \\
\hline Etheneimine & $\mathrm{H}_{2} \mathrm{CCNH}$ & $\lesssim 0.1$ & $\lesssim 2$ & & & & & \\
\hline Cyanoacetylene+ & $\mathrm{HC}_{3} \mathrm{NH}^{+}$ & $\lesssim 0.01$ & $\lesssim 0.2$ & & & & & \\
\hline Vinyl cyanide & $\mathrm{C}_{2} \mathrm{H}_{3} \mathrm{CN}$ & $\lesssim 0.01$ & $\lesssim 0.2$ & & & & & \\
\hline Ethyl cyanide & $\mathrm{C}_{2} \mathrm{H}_{5} \mathrm{CN}$ & $\lesssim 0.02$ & $\lesssim 0.7$ & & & & & \\
\hline Aminoacetonitrile & $\mathrm{H}_{2} \mathrm{NCH}_{2} \mathrm{CN}$ & $\lesssim 0.03$ & $\lesssim 0.7$ & & & & & \\
\hline Cyanopropyne & $\mathrm{CH}_{3} \mathrm{C}_{3} \mathrm{~N}$ & $\lesssim 0.002$ & $\lesssim 0.07$ & & & & & \\
\hline n-Propyl cyanide & $\mathrm{n}-\mathrm{C}_{3} \mathrm{H}_{7} \mathrm{CN}$ & $\lesssim 0.05$ & $\lesssim 0.8$ & & & & & \\
\hline Cyanopentadiyne & $\mathrm{CH}_{3} \mathrm{C}_{5} \mathrm{~N}$ & $\lesssim 0.01$ & $\lesssim 0.3$ & & & & & \\
\hline
\end{tabular}

Note: The first two columns report the species name and formula. Third, fourth and fifth columns report the values of the inner and outer abundances $X_{\text {in }}$ and $X_{\text {out }}$ (with respect to $\mathrm{H}_{2}$ ), and $T_{j u m p}$. Columns 6 and 7 report the degrees of freedom and the minimum reduced $\chi^{2}$. The last two columns report the radius and the sizes (diameter) at which the abundance jump occurs. The error bars are at $2 \sigma$ level confidence. The top half table lists the detected species, the bottom half table the upper limit on the abundances of undetected COMs (see text). 

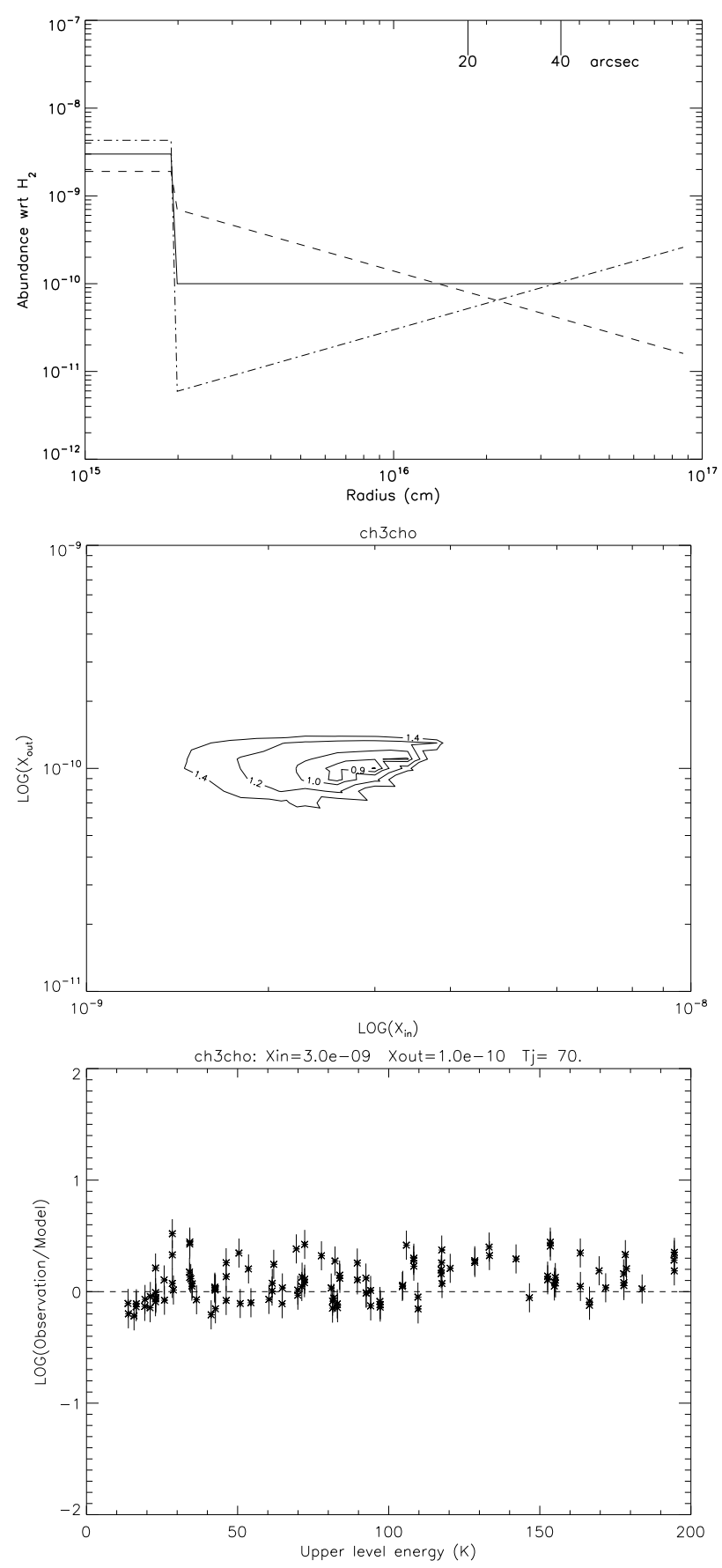

Fig. 1. - Example of the acetaldehyde analysis. Upper panel: Abundance profiles of the best fit obtained considering the cold envelope abundance profile following a power law dependence with radius (Eq. 1) of $\alpha$ equal to -1 (dashed), 0 (solid) and 1 (dotted-dashed) respectively. Middle panel: $\chi^{2}$ contour plot assuming the best fit $T_{\text {jump }}=70 \mathrm{~K}$ and $\alpha=0$. Bottom panel: Ratio of the observed over predicted line flux as a function of the upper level energy of the transition for the best fit solution (Table 1). 


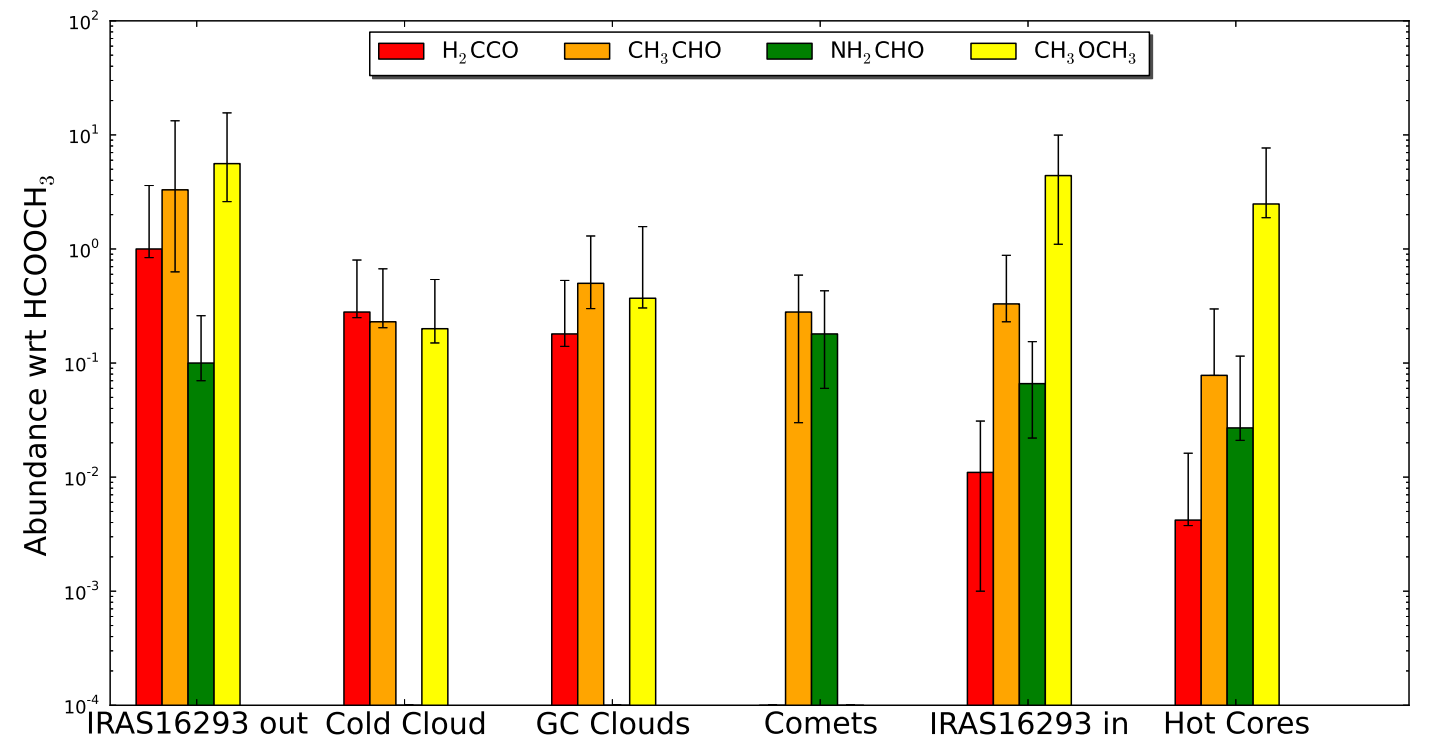

Fig. 2.- Abundances of the five COMs analysed in this work, normalised to the methyl formate abundance, in different objects: inner and outer envelope of IRAS16293 (this work), Cold Clouds (Bacmann et al. 2012; Cernicharo et al. 2012), Galactic Center (GC) Clouds (Requena-Torres et al. 2006, 2008), Hot Cores (Gibb et al. 2000; Ikeda et al. 2001; Bisschop et al. 2007: note that we did not include SgrB2 in this sample), and Comets (Mumma \& Charnley 2011). Error bars represent the dispersion in each group of objects, except IRAS16293 for which error bars reflect the errors in the abundance determination (Tab. 1). 

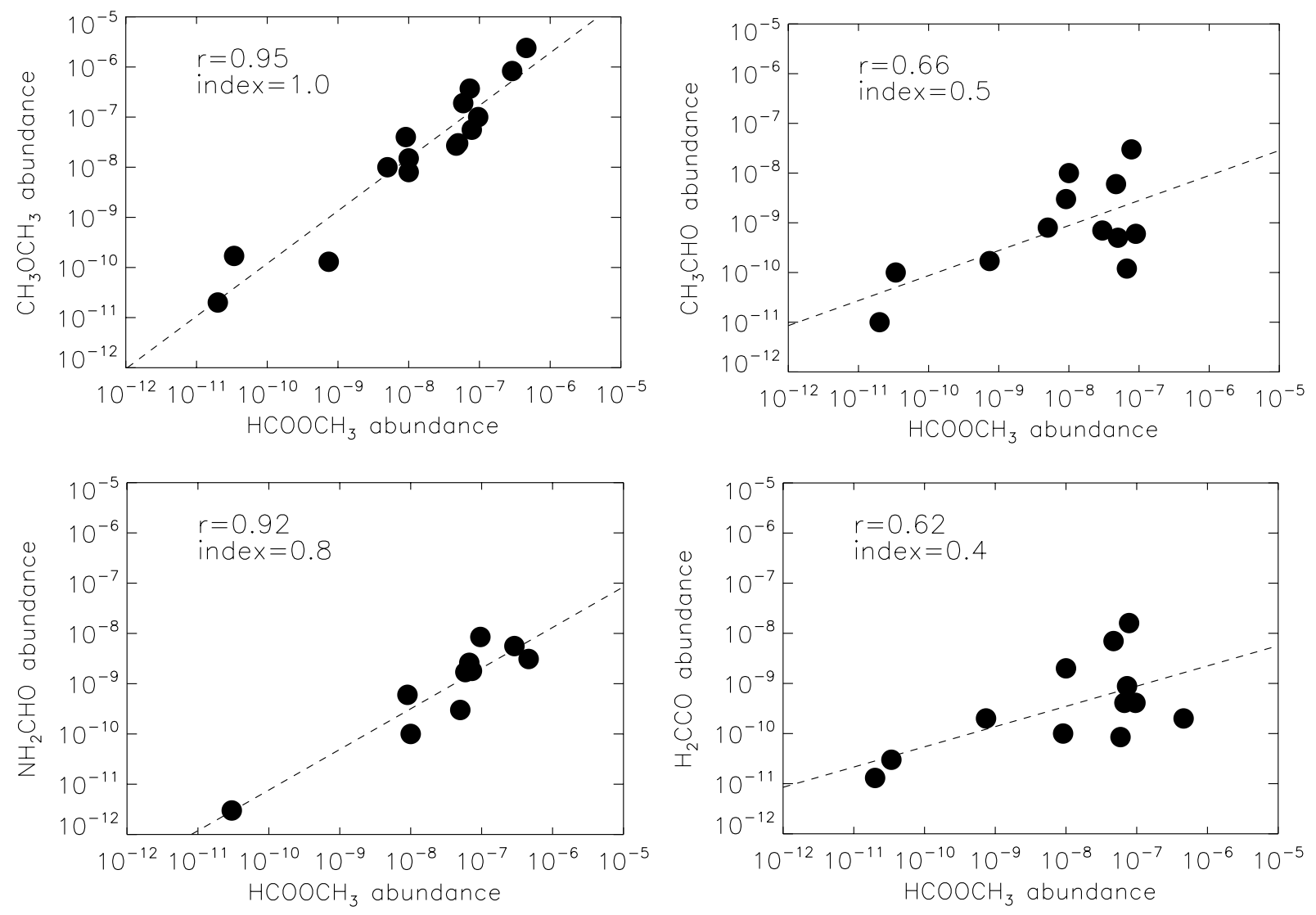

Fig. 3.- Abundance of dimethyl ether (top left), formamide (bottom left), acetaldehyde (top right) and ketene (bottom right) as a function of the abundance of methyl formate in different ISM sources. The correlation coefficient $r$ and the power law index are reported for each species. 